\title{
Studies toward Synthesis of Arteminolides: Intramolecular [5+2] Oxidopyrylium Ion Cycloaddition Reactions with Silicon Tether
}

\author{
Jeong-Hun Sohn \\ Institut Pasteur Korea, Seongnam 463-400 Korea. E-mail: sohnjh@ip-korea.org \\ Received May 30, 2010, Accepted May 31, 2010
}

Key Words: Oxidopyrylium ion, [5+2] Cycloaddition reaction, Vinylsilane, Arteminolide

Arteminolides A-D (1-4) are sesquiterpene lactones isolated from the leaves of Artemisia sylvatica Maxim and have been reported to be the strongest inhibitors of farnesyltransferase (FTase) ${ }^{1}$ with good selectivity from natural sources. ${ }^{2}$ While the biological profile and structural complexity of these natural products are intriguing, there has been no report on the synthesis of these natural products since their first isolation in 1998 . $^{3}$

The most logical precursors for the total synthesis of arteminolides are dehydromatricarin (5) and $\mathbf{6}$, since they are believed to be the biogenic precursors of arteminolides through Diels-Alder reaction ${ }^{4}$ (Scheme 1). Identification of enone 5 and diene $\mathbf{6}$ along with studies on the related natural products strongly support this route. ${ }^{4 \mathrm{~b}}$ We envisioned that the two precursors, $\mathbf{5}$ and $\mathbf{6}$, could be obtained from the intermediates $\mathbf{7}$ and $\mathbf{8}$, respectively, through a sequence including Pauson-Khand reaction.
The intermediates $\mathbf{7}$ and $\mathbf{8}$ could be prepared from a common precursor $\mathbf{9}$, in which the silicon was incorporated to serve as surrogates for both hydroxyl group and hydrogen corresponding to $\mathrm{C} 8-\mathrm{OH}$ of $\mathbf{5}$ and $\mathrm{C} 8$ '-H of $\mathbf{6}$, respectively. ${ }^{5}$ The ether bridge of 9 would allow selective induction of stereochemistry at C6 and $\mathrm{C} 8$, and C6' in turn could be obtained through an intramolecular [5+2] cycloaddition reaction of oxidopyrylium ion ${ }^{6}$ with vinylsilyl ether $\mathbf{1 0}$ as the key step since we observed that the cycloaddition reaction with silylated olefin proceeded well. ${ }^{3 b}$

The synthesis of the common intermediate 9 commenced with selective mono-silylation of 1,3-propanediol (11), followed by Swern oxidation, resulting in corresponding aldehyde which was then coupled with lithiated furan to afford furanyl alcohol 12 in 55\% yield for three steps (Scheme 2). The TBS group was replaced by vinylsilyl group via removal of TBS using TBAF
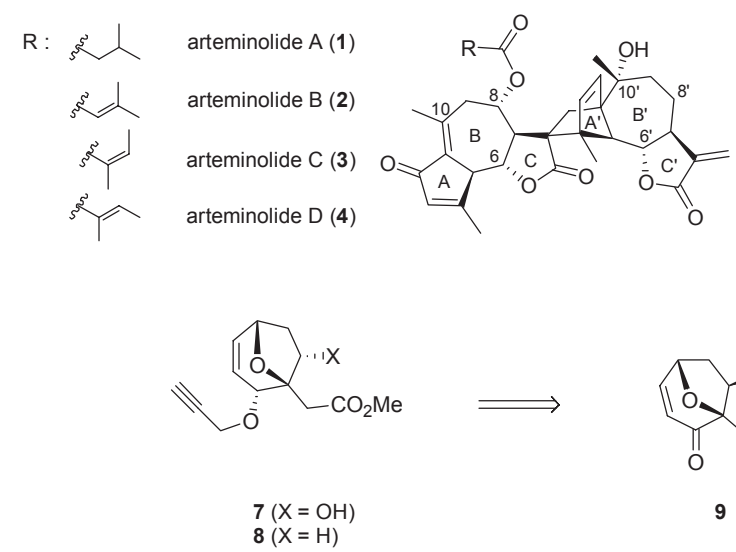

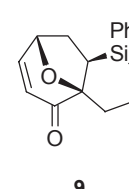

Diels-Alder reaction
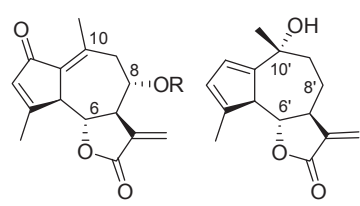

dehydromatricarin A (5)

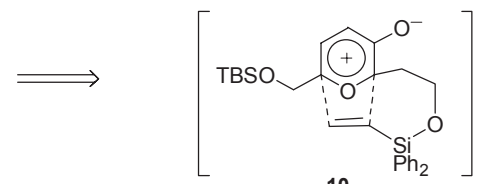

Scheme 1. Structure of arteminolides A-D (1-4) and their retrosynthetic analysis

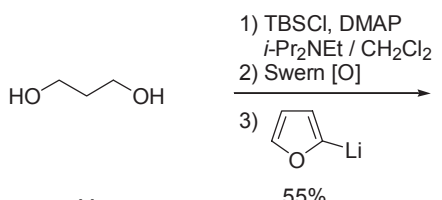

11

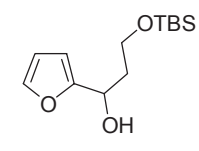

12

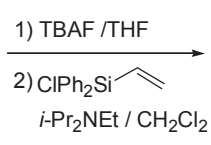

$70 \%$

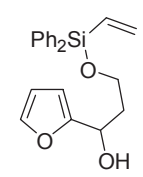

13

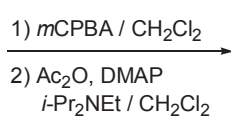

$73 \%$<smiles>C=C[SnH2]c1ccccc1</smiles>

14

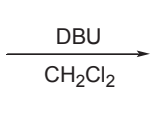

$\stackrel{\mathrm{H}}{\mathrm{Cl}_{2}}$

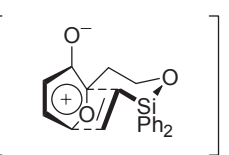

15

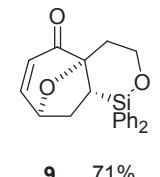

ingle diastereomer

Scheme 2. Synthesis of the common intermediate 9 

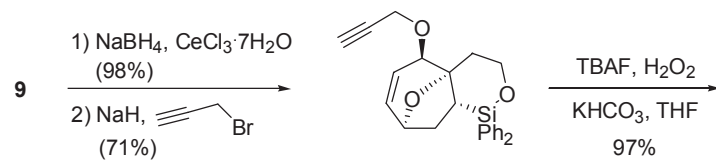

16
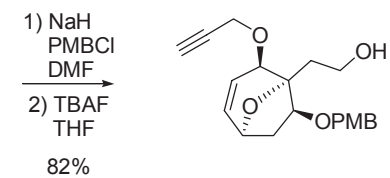

20

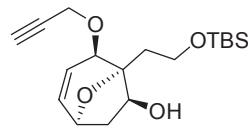

19
$82 \%$

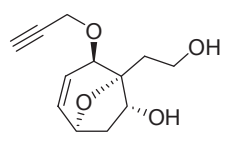

17

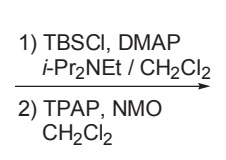

$81 \%$

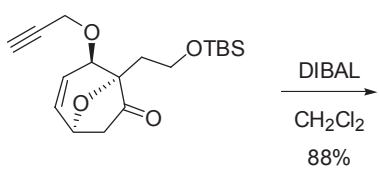

18

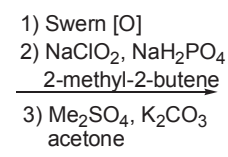

$65 \%$

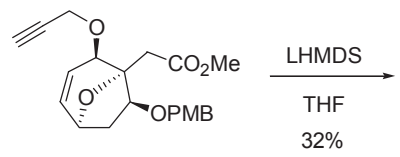

21

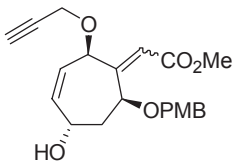

22

single diastereomer

Scheme 3. Transformation of 9 to 21

generating corresponding diol, followed by selective coupling of the primary hydroxyl group with the vinylsilyl chloride in the presence of $i-\operatorname{Pr}_{2} \mathrm{NEt}$ to produce vinylsilyl ether $\mathbf{1 3}$ in 70\% yield. As a precursor to the oxidopyrylium ion, pyran 14 was prepared in two-steps including oxidation-rearrangement of 13 using $m$ CPBA in $\mathrm{CH}_{2} \mathrm{Cl}_{2}$, followed by acetylation of the resulting secondary hydroxyl group using $\mathrm{Ac}_{2} \mathrm{O}$ and DMAP in the presence of $i-\operatorname{Pr}_{2} \mathrm{NEt}$. Treatment of pyran 14 in methylene chloride with DBU generated the oxidopyrylium ion, and in situ cycloaddition reaction proceeded well to give the desired product 9 as a single diastereomer ${ }^{8}$ in $71 \%$ yield via a transition state $\mathbf{1 5}$, in which the cycloaddition occurs with favorable chair form of six-membered ring. Through this route, we could synthesize the desired common skeleton of the B- and B'-rings of arteminolides in eight-steps and $20 \%$ overall yield from the propanediol 11.

Since the C8-OR in B-ring determined the identity of the activity of ateminolides ${ }^{1}$ and the replacement of the silicon in 9 with hydrogen was easily done under the conventional conditions for removal of silyl, we focused on the transformation of 9 into functionalized B-ring. The ether bridge of 9 allowed stereoselective reduction of the carbonyl, which resulted in the corresponding alcohol in high yield as a single diastereomer with the hydroxyl group trans to the silyl group. ${ }^{8}$ The generated hydroxyl group was converted to propargyl ether $\mathbf{1 6}$ for the construction of A-ring through Pauson-Khand reaction as well as protection of the hydroxyl group. Treatment of $\mathbf{1 6}$ with Tamao oxidation conditions using $\mathrm{KF}, \mathrm{H}_{2} \mathrm{O}_{2}$ and $\mathrm{KHCO}_{3}$ in THF$\mathrm{MeOH}^{5 \mathrm{a}}$ gave diol 17 in only $20 \%$ yield but, instead, the modified conditions using TBAF, $\mathrm{H}_{2} \mathrm{O}_{2}$ and $\mathrm{KHCO}_{3}$ in THF increased the yield to $97 \%$. After protection of the primary hydroxyl group in $\mathbf{1 7}$ as TBS ether the remaining secondary hydroxyl group was epimerized through oxidation using TPAP-NMO, followed by reduction using DIBAL to afford alcohol 19, in which the hydroxyl group and the propargyl ether are at desired cis conformation. Protection of the hydroxyl group as PMB ether was followed by removal of TBS to give primary alcohol 20. Treatment of $\mathbf{2 0}$ with Swern oxidation conditions afforded the resulting aldehyde, which was then oxidized using $\mathrm{NaClO}_{2}$ and $\mathrm{NaH}_{2} \mathrm{PO}_{4}$, and methylated using $\mathrm{Me}_{2} \mathrm{SO}_{4}$ to afford ester 21 in $65 \%$ yield. The ether bridge had to be removed for the form- ation of the five-membered A-ring stereoselectively through Pauson-Khand reaction since the direct Pauson-Khand reaction of 21 would produce the opposite stereo-isomer to $\mathbf{6}$ at the ring junction. Base-mediated cleavage of the ether bridge with LHMDS successfully cleaved the ether bridge to afford 22 as a single diastereomer (Scheme 3 ).

In summary, we prepared compound 9 which has the common ring skeleton of the B- and B'-rings of arteminolides in eightsteps and $20 \%$ overall yield from the 1,3-propanediol, through a route that featured an unprecidented intramolecular [5+2] oxidopyrylium cycloaddition reaction with vinylsilyl ether tether as the key step. Studies directed toward the completion of the synthesis of arteminolides through the stereoselective PausonKhand reaction are currently underway.

Acknowledgments. This work was supported by Korean Ministry of Education, Science and Technology.

\section{Reference}

1. (a) Lee, S.-H.; Kim, M.-J.; Bok, S. H.; Lee, H.; Kwon, B. -M. J. Org. Chem. 1998, 63, 7111. (b) Lee, S.-H.; Kang, H.-M.; Song, H.-C.; Lee, H.; Lee, U. C.; Son, K.-H.; Kim, S.-H.; Kwon, B.-M. Tetrahedron 2000, 56, 4711. (c) Lee, S.-H.; Kim, H.-K.; Seo, J.-M.; Kang, H.-M.; Kim, J.-H.; Son, K.-H.; Lee, H.; Kwon, B.-M.; Shin, J.; Seo, Y. J. Org. Chem. 2002, 67, 7670. (d) Lee, S.-H.; Lee, M.-Y.; Kang, H.-M.; Han, D. C.; Son, K.-H.; Yang, D. C.; Sung, N.-D.; Lee, C. W.; Kim, H. M.; Kwon, B.-M. Bioorg. Med. Chem. 2003, 11, 4545 .

2. Agrawal, A. G.; Somani, R. R. Mini-Rev. Med. Chem. 2009, 9, 638.

3. Our studies on the synthesis of arteminolides: (a) Lee, H.-Y.; Sohn, J.-H.; Kim, H. Y.; Kwon, B.-M. Tetrahedron Lett. 2001, 42, 1695. (b) Sohn, J.-H. Bull. Korean Chem. Soc. 2009, 30, 2517.

4. (a) Jakupovic, J.; Chem, Z.-L.; Bohlmann, F. Phytochemistry 1987, 26, 2777. (b) Bohlman, F.; Zdero, C. Phytochemistry 1978, 17, 1595. (c) Zhang, W.; Luo, S.; Fang, F.; Chen, Q.; Hu, H.; Jia, X.; Zhai, H. J. Am. Chem. Soc. 2005, 127, 18. (d) Wong, H.-F.; Brown, G. D. J. Nat. Prod. 2002, 65, 481.

5. (a) Tamao, K.; Kawachi, A.; Tanaka, Y.; Ohtani, H.; Ito, Y. Tetrahedron 1996, 52, 5765. (b) Fleming, I. Chem. Rev. 1997, 97, 2063.

6. Katritzky, A. R. Chem. Rev. 1989, 89, 827.

7. Lefebvre, Y. Tetrahedron Lett. 1972, 13, 133.

8. Stereochemistries were determined through H-H COSY or NOE experiments. 